\title{
Pseudospin lifetime in relaxed-shape armchair graphene nanoribbons due to in-plane phonon modes
}

\author{
Sanjay Prabhakar, ${ }^{1, *}$ Roderick Melnik, ${ }^{1,2}$ and Luis Bonilla ${ }^{3}$ \\ ${ }^{1}$ The MS2Discovery Interdisciplinary Research Institute, $M^{2} N e T$ Laboratory, Wilfrid Laurier University, Waterloo, ON N2L 3C5, Canada \\ ${ }^{2}$ BCAM-Basque Center for Applied Mathematics, E48009 Bilbao, Spain \\ ${ }^{3}$ Gregorio Millan Institute, Fluid Dynamics, Nanoscience and Industrial Mathematics, \\ Universidad Carlos III de Madrid, 28911 Leganes, Spain
}

(Received 10 December 2015; revised manuscript received 17 February 2016; published 11 March 2016)

\begin{abstract}
We study the influence of ripple waves on the band structures of strained armchair graphene nanoribbons. We argue that the Zeeman pseudospin (p-spin) splitting energy induced by ripple waves might not be neglected for smaller widths of armchair graphene nanoribbons (GNRs). We show that the p-spin splitting energy breaks the symmetry of degeneracy due to the ripple-induced Zeeman effect in GNRs, originating from electromechanical coupling. We estimate the p-spin lifetime in strained armchair GNRs caused by in-plane phonon modes for possible applications in straintronics and quantum information processing. By considering higher order terms in the strain tensor expansion, we also demonstrate that highly asymmetric band structures of GNRs induce asymmetric phonon-mediated p-spin relaxation. Such asymmetric p-spin relaxation is not possible for unstrained armchair and zigzag GNRs. In particular, we report that the p-spin transition rate decreases like $B_{0}^{5}$ (as a function of p-magnetic fields), $L^{-9}$ (as a function of GNR width) and $\tau_{e}^{-1}$, where $\tau_{e}$ is the externally applied tensile edge stress.
\end{abstract}

DOI: 10.1103/PhysRevB.93.115417

\section{INTRODUCTION}

Graphene, just one atom thick, is purely a two-dimensional material, and atoms spread throughout the sample of the graphene sheet in the form of a honeycomb lattice, which is of potential interest for developing next-generation optoelectronic devices. At room temperature, electrons that possess the properties of massless Dirac fermions can travel ballistically over several micrometers without scattering. Researchers have shown that transistors made of graphene can operate at a speed two times faster than that of the best silicon transistors of similar size. The massless Dirac fermions in graphene also provide an opportunity for researchers to study novel quantum mechanical properties with current state-of-the-art tools. For example, measurements of quantum mechanical properties such as the half-integer quantum Hall effect, nonzero Berry phase, conductivity of electrons, and high-mobility charge carriers (100 times that of silicon) may reaffirm graphene as a promising material for making next-generation optoelectronic, spintronic, and straintronic devices [1-6]. Potential applications of graphene in photonics and optoelectronics devices (solar cells, light-emitting devices for touching screens, photodetectors, and ultrafast lasers) have been discussed in Ref. [7], where the combination of its unique optical and electronic properties is explored, even in the absence of any bandgap. The authors explore how a linear dispersion of the Dirac electrons enables ultrawide band tunability. Experimental and theoretical studies of spin initialization and readout, defect-induced magnetic moments, spin-orbit coupling, and spin relaxation in graphene are also presented in Refs. [8] and [9]. Further experimental and theoretical study in graphene spintronics will indeed require addressing the development of applications such as spin transistors and spin logic devices, as well as utilizing

\footnotetext{
*sprabhakar@wlu.ca
}

more exotic physical properties including topological states and proximity-induced phenomena in graphene and other two-dimensional materials [8-10]. The pseudomorphic vector potential in graphene may induce large pseudomagnetic fields (10 times larger than the largest steady-state magnetic field ever achieved in a laboratory) [6,11]. Electrons under such large pseudomagnetic fields, which have not been investigated experimentally so far, may provide a new level of physics.

In-plane or out-of-plane ripple waves in graphene may be induced by applying tensile edge stress (relaxed-shape graphene) or adsorbed hydro-oxide molecules throughout the sample of the graphene sheet [12-15]. Strain fields, dislocations, and defects can also be utilized to control the band structures of graphene [16,17]. The influence of such effects (ripple, strain fields, dislocations, defects) on the band structure of graphene can be studied by coupling a strain tensor originating from electromechanical effects through a pseudopotential $[18,19]$. This pseudopotential induces large pseudomagnetic fields (300 to $1000 \mathrm{~T}$ ) [11,20]. This is linked to purely intrinsic properties and may be useful for designing optoelectronic devices for straintronic applications. It is assumed that graphene can easily sustain such large pseudomagnetic fields because it is one of the strongest materials (intrinsic breaking strength of $\approx 42 \mathrm{~N} / \mathrm{m}, 100$ times stronger than steel [6]) found in nature, which is confirmed experimentally in Ref. [21]. The strain tensor may also induce bandgaps at the Dirac point, which may open new opportunities for making next-generation optoelectronic and quantum information processing devices. In this paper, we show that strain engineering can be used to tune graphene's band structure in ways that may be useful for taking advantage of quantum confinement effects (to create dot and ribbon geometries). In particular, we develop a new theoretical approach to estimate the phonon-mediated p-spin lifetime in strained armchair GNRs for application in quantum information processing, among other areas of applications. 
The paper is organized as follows. In Sec. II, we provide a theoretical description of coupling between the electromechanical effects and the band structures of GNRs and show that the electronic properties of strained GNRs are highly asymmetric. Here we show that the pseudomagnetic fields originating from the pseudomorphic vector potential might induce large Zeeman p-spin splitting energies for smaller GNR widths, which provides evidence of broken p-spin symmetry of degeneracy. We also provide theoretical descriptions of orbital and $\mathrm{p}$-spin relaxation mechanisms caused by in-plane phonon modes for straintronic and quantum information processing. In addition to the results associated with the electromechanical effects and band structures of strained GNRs, in Sec. III we analyze p-spin relaxation rates vs several control parameters, such as the width of the GNRs and the applied tensile edge stress through the boundary and pseudomagnetic fields, and demonstrate that resonant peaks in the p-spin lifetime can be observed. Finally, we summarize our results in Sec. IV.

\section{THEORETICAL MODEL}

The total elastic energy density associated with the strain for a two-dimensional graphene sheet can be written as [25,26] $2 U_{s}=C_{i k l m} \varepsilon_{i k} \varepsilon_{l m}$. Here $C_{i k l m}$ is a tensor of rank 4 (the elastic modulus tensor) and $\varepsilon_{i k}$ (or $\varepsilon_{l m}$ ) is the strain tensor. In the above, we write the strain tensor components as $\varepsilon_{x x}=$ $\partial_{x} u_{x}, \varepsilon_{y y}=\partial_{y} u_{y}, \varepsilon_{x y}=\left(\partial_{y} u_{x}+\partial_{x} u_{y}\right) / 2$, where $u_{i}$ are the in-plane displacements [14]. The stress tensor components can be easily found from the expression $\sigma_{i k}=\partial U_{s} / \partial \varepsilon_{i k}$. In the continuum limit, elastic deformations of graphene sheets under external tension in the $x$ direction are described by the Navier equations $\partial_{k} \sigma_{i k}=F_{\mathrm{ext}}^{x} / t$, where $F_{\mathrm{ext}}^{x}=\tau_{e} q \cos (q x)$. Here $q=2 \pi / \lambda$ is the period length of the in-plane ripple waves, $\tau_{e}$ is the externally applied tensile edge stress, and $t=0.35 \mathrm{~nm}$ is the thickness of the single-layer graphene. We assume armchair GNRs elongated along the $y$ direction and apply tensile edge stress only along the $x$ direction (see Fig. 1), which only induces $\varepsilon_{x x}$ as a nonvanishing strain tensor component [27]. Assuming a vanishing displacement vector at $x= \pm L / 2$, we derive the expression for the displacement vector from the equilibrium Navier equations as

$$
u_{x}=\frac{\tau_{e}}{q C_{11} t}\left\{\cos \left(\frac{q L}{2}\right)-\cos (q x)\right\} .
$$

In Fig. 1, we have plotted the distribution of pseudomagnetic fields (middle) and strain tensor (right). Below we show that pseudofields induce high pseudo-Zeeman energies for smaller ribbon widths and the strain tensor induces the quantum confinement effect. By considering higher order terms in the expansion of $\sin x$ in the strain tensor, we also show that highly asymmetric band structures of GNRs are possible.

Now we turn to the study of the influence of the strain tensor on the electronic properties of GNRs. In the continuum limit, by expanding the momentum close to the $K$ point in the Brillouin zone, the Hamiltonian for $\pi$ electrons at the K point reads $H=H_{0}+H_{z}$ as [22]

$$
H=v_{F}\left(\sigma_{x} P_{x}+\sigma_{y} P_{y}\right)+g_{0} \mu_{B} B_{s} \sigma_{z} / 2,
$$
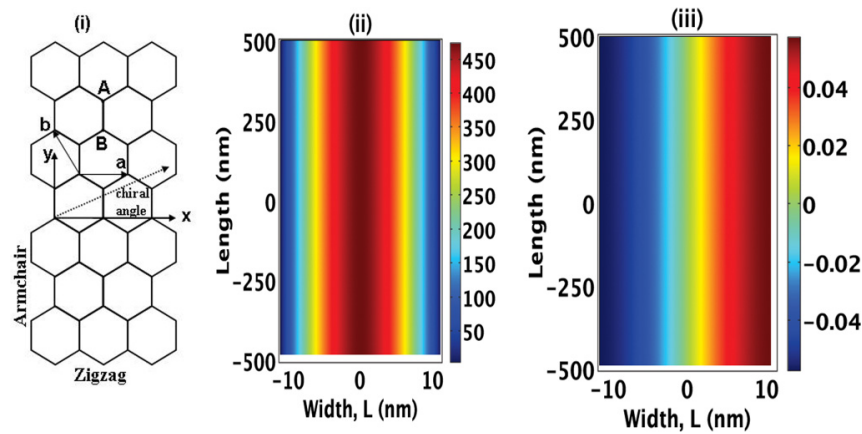

FIG. 1. (i) Schematic of the lattice structure of two-dimensional armchair graphene nanoribbons (GNRs). (ii) Spatial dependence of the induced pseudomagnetic field, in teslas (see color scale). (iii) Strain originating from electromechanical effects due to externally applied tensile edge stress in GNRs. Pseudomagnetic fields originating from electroelasticity induce Zeeman p-spin energy, while the strain tensor induces a quantum confinement potential that might be utilized in engineered straintronic devices. Here we chose $\tau_{e}=45 \mathrm{eV} / \mathrm{nm}, t=0.35 \mathrm{~nm}, L=3 \sqrt{3} \mathrm{Na}$, with $N=30$ and $1 \mu \mathrm{m}$ as the length of the ribbon, $C_{11}=2246.22 \mathrm{eV} / \mathrm{nm}^{2}$, and $\lambda=2 L$. See also Refs. [20,22-24].

where $P=p-\hbar A$, with $p=-i \hbar \partial_{x}$ being the canonical momentum operator and $\mathbf{A}=\beta\left(-2 \varepsilon_{x y}, \varepsilon_{y y}-\varepsilon_{x x}, 0\right) / a$ the vector potential induced by the pseudomorphic strain tensor [22,28]. Here $a$ is the lattice constant, $\beta=-\partial \ln \varsigma / \partial \ln a \approx 2$ is a dimensionless parameter that characterizes the coupling between Dirac electrons and lattice deformations, and $\varsigma$ is the nearestneighbor hopping parameter. The last term on the right-hand side of (2) is the Zeeman energy due to p-spin, where $B_{s}=$ $\nabla \times A=-B_{0} \cos (q x) \hat{z}$ with $B_{0}=2 \pi \hbar \beta \tau_{e} q / e C_{11} t a$ is the effective pseudomagnetic field induced by the pseudomorphic vector potential. The Zeeman term due to $\mathrm{p}$-spin is usually neglected because it is assumed that the induced p-spin due to ripple waves is not able to break the symmetry of degeneracy in the band structure of graphene nanoribbons (GNRs). However, in this paper for narrower GNRs, by applying tensile edge stress through the boundary of the armchair edge along the $x$ direction [see Fig. 1(a)], we show that a giant Zeeman p-spin splitting energy due to ripple waves can break the symmetry of degeneracy, which can be utilized in designing straintronic devices. In the above expression for the pseudomagnetic field, $B_{s}$, we see that the magnitude of the pseudomagnetic field amplitude, $B_{0}$, is directly proportional to the period length, $q=2 \pi / \lambda$, of the in-plane ripple waves. Assuming $\lambda \approx L$, we can write $B_{0} \propto 1 / L$. This means that the magnitude of the pseudomagnetic field amplitude, $B_{0}$, is enhanced for smaller GNR widths. It is this effect that is responsible for inducing a giant Zeeman p-spin energy. Hence, we can confirm that the Zeeman $\mathrm{p}$-spin energy, originating from electroelasticity due to externally applied tensile edge stress that induces a nonvanishing strain tensor, is responsible for breaking the symmetry of degeneracy in smaller-width GNRs [see second and third terms on the right-hand side of Eq. (17)]. Note that the contribution of the second and third terms on the right-hand side of Eq. (17) to the total energy eigenvalues becomes extremely small for either wider GNRs or smaller values of externally applied stress, $\tau_{e}$. Thus, narrower GNRs and high 
externally applied tensile stresses are the key requirements in our case to break the symmetry of degeneracy.

We assume $H \psi=\varepsilon \psi$, where $\psi\left(\psi_{A}\right.$ and $\psi_{B}$ are its components) is the spinor wave function of Hamiltonian (2). In Fig. 1(i), we assume that the edges of the graphene nanoribbons are parallel to the $y$ axis. In this case, the translational symmetry guarantees that this spinor wave function can be written as [2]

$$
\psi(r)=\exp \left(i k_{y} y\right)\left(\begin{array}{c}
\phi_{A}(x) \\
\phi_{B}(x)
\end{array}\right),
$$

where $\phi_{A}(x)$ and $\phi_{B}(x)$ are the wave functions of quantized graphene electrons on sublattices $\mathrm{A}$ and $\mathrm{B}$, respectively. Thus from (2), we write the two coupled equations as

$$
\begin{aligned}
& \frac{1}{2} g_{0} \mu_{B} B_{s} \phi_{A}+\hbar v_{F}\left(-i \partial_{x}-i k_{y}-i \frac{\beta}{a} \varepsilon_{x x}\right) \phi_{B}=\varepsilon \phi_{A}, \\
& \hbar v_{F}\left(-i \partial_{x}+i k_{y}+i \frac{\beta}{a} \varepsilon_{x x}\right) \phi_{A}-\frac{1}{2} g_{0} \mu_{B} B_{s} \phi_{B}=\varepsilon \phi_{B} .
\end{aligned}
$$

Exact solutions of (4) and (5) are nontrivial. Thus, we seek to apply perturbation theory to get some insight into the behaviors of the band structures of strained GNRs. First, we assume $B_{s}=$ $-B_{0} \cos (q \hat{x}) \approx-B_{0}\left(1-q^{2} \hat{x}^{2} / 2 !+q^{4} \hat{x}^{4} / 4 !\right)$ and write the total Hamiltonian $H=\tilde{H}_{0}+\tilde{H}_{z}\left(\hat{x}^{2}, \hat{x}^{4}\right)$, where

$$
\begin{gathered}
\tilde{H}_{0}=v_{F}\left(\sigma_{x} p_{x}+\sigma_{y} \hbar k_{y}-\hbar \sigma_{y} A_{y}\right)+\Delta \sigma_{z} / 2, \\
\tilde{H}_{z}=-\Delta q^{2} \hat{x}^{2} \sigma_{z} / 4+\Delta q^{4} \hat{x}^{4} \sigma_{z} / 48,
\end{gathered}
$$

and $\Delta=-g_{0} \mu_{B} B_{0}$. Note that the coefficient of $\sigma_{z}$ in (6) does not depend on the position operator and it might be impossible to break the p-spin symmetry of degeneracy. However, (7) depends on the position operator and below we show that the first-order energy correction term of $\tilde{H}_{0}$, due to $\tilde{H}_{z}$, breaks the symmetry of degeneracy, which can be utilized to induce p-spin splitting of electron-hole-like states for straintronic applications of GNRs. The second-order energy correction term of $\tilde{H}_{0}$ due to $\tilde{H}_{z}$ also provides highly asymmetric electron-hole $\mathrm{p}$-spin relaxation in GNRs mediated by in-plane phonon modes.

We assume $\tilde{H}_{0} \psi=\tilde{\varepsilon} \psi$ and write two coupled equations associated with (6) as

$$
\begin{aligned}
& -i \hbar v_{F}\left(\partial_{x}+k_{y}+\beta \varepsilon_{x x} / a\right) \phi_{B}=(\tilde{\varepsilon}-\Delta / 2) \phi_{A}, \\
& -i \hbar v_{F}\left(\partial_{x}-k_{y}-\beta \varepsilon_{x x} / a\right) \phi_{A}=(\tilde{\varepsilon}+\Delta / 2) \phi_{B} .
\end{aligned}
$$

Now we apply the operator $-i \hbar v_{F}\left(\partial_{x}-k_{y}-\beta \varepsilon_{x x} / a\right)$ from the left-hand side of (8) and the operator $-i \hbar v_{F}\left(\partial_{x}+k_{y}+\beta \varepsilon_{x x} / a\right)$ from the left-hand side of (9) and write a single decoupled second-order partial differential equation as

$$
\begin{aligned}
& \left(\hbar v_{F}\right)^{2}\left(-\partial_{x}^{2}+2 \frac{\beta}{a} \varepsilon_{x x} k_{y} \mp \frac{\beta}{a}\left[\partial_{x}, \varepsilon_{x x}\right]+k_{y}^{2}+\frac{\beta^{2}}{a^{2}} \varepsilon_{x x}^{2}\right) \phi_{B, A} \\
& =\left(\tilde{\varepsilon}^{2}-\Delta^{2} / 4\right) \phi_{B, A} .
\end{aligned}
$$

By utilizing the identity $\left[x^{\tilde{n}}, \partial_{x}\right]=-\tilde{n} x^{\tilde{n}-1}$ with $\tilde{n}=$ $1,2,3, \ldots$ and by considering $\varepsilon_{x x} \approx \tau_{e}\left(q x-q^{3} x^{3} / 3 !\right) / C_{11} t$, we formulate (10) as

$\left[\frac{p_{x}^{2}}{2 m_{0}}+\frac{1}{2} m_{0} \omega_{0}^{2} x^{2}+\tilde{A}\left(x-\frac{q^{2} x^{3}}{3 !}\right)\right] \phi_{B, A}=E \phi_{B, A}$,

where

$$
\begin{aligned}
& E=\frac{\tilde{\varepsilon}^{2}-A}{2 m_{0} v_{F}^{2}}, \quad \omega_{0}=\frac{\hbar}{m_{0}}\left[\left(\frac{\beta \tau_{e} q}{C_{11} t a}\right)^{2} \pm \frac{\beta \tau_{e} q^{3}}{2 C_{11} t a}\right]^{1 / 2}, \\
& \tilde{A}=\frac{\hbar^{2} \beta \tau_{e} q k_{y}}{m_{0} C_{11} t a}, \quad A=\left(\hbar v_{F}\right)^{2}\left(k_{y}^{2} \mp \frac{\beta \tau_{e} q}{C_{11} t a}\right)+\frac{\Delta^{2}}{4} .
\end{aligned}
$$

We treat $\tilde{A}\left(x-q^{2} x^{3} / 3\right.$ ! $)$ as a perturbation and write the total energy eigenvalues associated with the Hamiltonian $\tilde{H}_{0}$ as

$$
\tilde{\varepsilon}_{n}= \pm\left[2 m_{0} v_{F}^{2}\left\{\left(n+\frac{1}{2}\right) \hbar \omega_{0}+\varepsilon_{n}^{(2)}\right\}+A\right]^{1 / 2},
$$

where

$$
\begin{gathered}
\varepsilon_{n}^{(2)}=\sum_{m \neq n} \frac{\left|\left\langle m\left|\tilde{A} \hat{x}-\tilde{A} q^{2} \hat{x}^{3} / 3 !\right| n\right\rangle\right|^{2}}{\varepsilon_{n}^{0}-\varepsilon_{m}^{0}} \\
\left\langle m\left|\hat{x}-q^{2} \hat{x}^{3} / 3 !\right| n\right\rangle \\
=\sqrt{\frac{\hbar}{2 m_{0} \omega_{0}}\left(\sqrt{n} \delta_{m, n-1}+\sqrt{n+1} \delta_{m, n+1}\right)} \\
-\frac{q^{2}}{3 !}\left(\frac{\hbar}{2 m_{0} \omega_{0}}\right)^{3 / 2}\left(3 \sqrt{n^{3}} \delta_{m, n-1}+3 \sqrt{(n+1)^{3}} \delta_{m, n+1}\right. \\
+\sqrt{n(n-1)(n-2)} \delta_{m, n-3} \\
\left.+\sqrt{(n+1)(n+2)(n+3)} \delta_{m, n+3}\right),
\end{gathered}
$$

and $\varepsilon_{n}^{0}=(n+1 / 2) \hbar \omega_{0}$. Finally, by treating $\tilde{H}_{z}$ as a perturbation of $\tilde{H}_{0}$, we write the total energy eigenvalue of $H$ for strained GNRs as

$$
\begin{aligned}
\varepsilon_{n, \sigma_{z}}= & \tilde{\varepsilon}_{n}-\Delta \tilde{\xi}(2 n+1) \sigma_{z}+\Delta \tilde{\xi}^{2}\left(2 n^{2}+2 n+1\right) \sigma_{z} \\
& +\frac{\Delta^{2} \tilde{\xi}^{2}}{\tilde{\varepsilon}_{n}-\tilde{\varepsilon}_{m}}\left(\mid \sqrt{(n+1)(n+2)} \delta_{m, n+2}\right. \\
& \left.+\sqrt{n(n-1)} \delta_{m, n-2}+\left.(2 n+1) \delta_{m, n}\right|^{2}\right),
\end{aligned}
$$

where $\tilde{\xi}=q^{2} \hbar / 8 m_{0} \omega_{0}$. Evidently, the first-order energy correction term in (17) gives us the p-spin splitting of the energy bands of GNRs due to the applied tensile edge stress. Assuming $N=10$ and $\tau_{e}=45 \mathrm{eV} / \mathrm{nm}$ and utilizing (17), we find the p-spin splitting energy difference $\varepsilon_{0, \downarrow}-\varepsilon_{0, \uparrow}=25.3 \mathrm{meV}$ for sublattice $A$ and $19.3 \mathrm{meV}$ for sublattice $B$. This p-spin splitting energy due to ripple waves is much higher than the energy induced by the spin-orbit coupling effect $\left(10^{-3}\right.$ to $2.5 \mathrm{meV}$ ) [29] in GNRs. This indicates that the symmetry of p-spin degeneracy is broken due to ripple waves. Thus, the Zeeman energy term due to p-spin in the total Hamiltonian of strained GNRs cannot be ignored for narrower ribbons. In Fig. 2(a), we plot the dispersion relation $E(k)$ of GNRs. Clearly, the band structures of GNRs of sublattices A and B are different [the energy difference between $\mathrm{p}$-spin-up and p-spin-down states for sublattice $A$ is larger than that for sublattice B; see Fig. 2(b)], which may lead to asymmetric phonon-mediated $\mathrm{p}$-spin relaxation of electrons in sublattices 


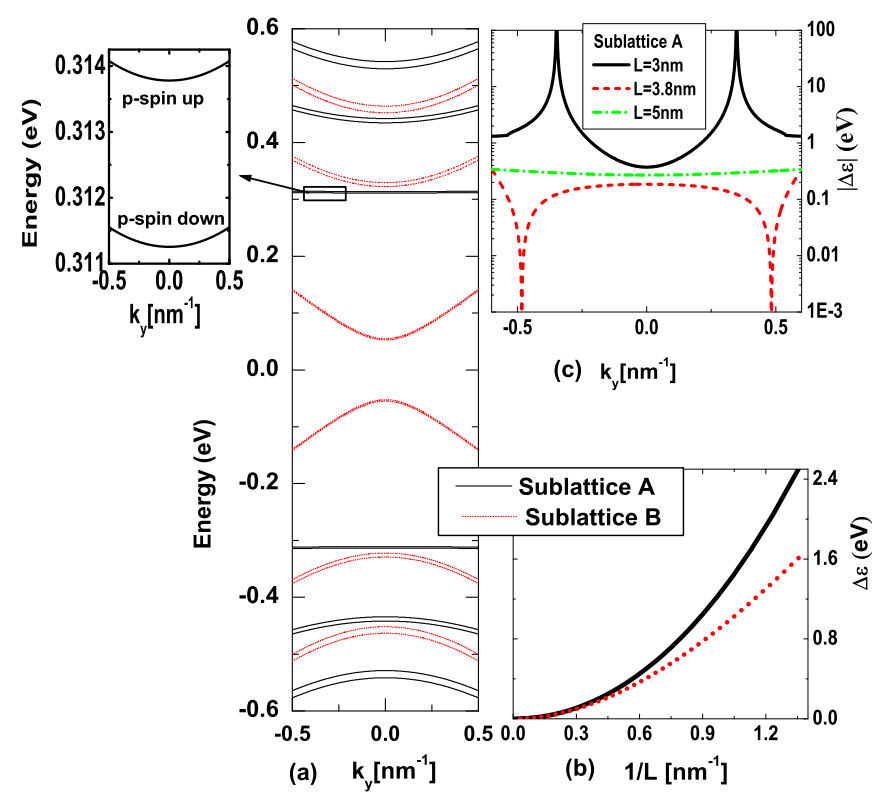

FIG. 2. Band structures of strained GNRs. (a) The case $L=$ $3 \sqrt{3} a N$ with $N=30$ clearly shows the p-spin splitting of several states. (b) The p-spin splitting energy difference is demonstrated to be enhanced in narrower GNRs at the Dirac point. It is also larger for sublattice A than B. (c) Cusp-like structures for $L=3 \mathrm{~nm}$ and dips, i.e., inverted cusp-like structures, for $L=3.8 \mathrm{~nm}$ are seen due to level crossing (see the text) of the energy bands. Here we chose $\tau_{e}=45 \mathrm{eV} / \mathrm{nm}$ in (a) and (c) and $\tau_{e}=250 \mathrm{eV} / \mathrm{nm}$ in (b). Other parameters were chosen as $a=0.142 \mathrm{~nm}, C_{11}=2246.22 \mathrm{eV} / \mathrm{nm}^{2}$, and $\lambda=2 L$.

A and B. For strained GNRs, $\omega_{0} \neq 0$ in Eq. (11) or $\varepsilon_{x x} \neq 0$ in Eqs. (8) and (9) may lead to a quantum confinement effect. In Fig. 2(c), we plot the energy difference between the ground state, with p-spin up, and the first excited state, with p-spin down, vs $k_{y}$. Here we see that at smaller ribbon widths, where the Zeeman energy due to p-spin is significantly higher, there is a crossing of the band structures that provides either dips (dashed line) or cusp-like (solid line) structures. For larger GNR widths (dashed-dotted line), the ripple-induced Zeeman energy becomes weaker and its influence on the symmetry of degeneracy is neglected. For unstrained armchair and zigzag GNRs, it is impossible to find band crossing.

\section{RESULTS AND DISCUSSION}

We now turn to the calculation of $\mathrm{p}$-spin relaxation induced by in-plane phonon modes due to externally applied tensile edge stress along the shorter edge in GNRs. The coupling between electron and phonon with mode $\mathbf{k} \alpha$ (k is the phonon wave vector, and the branch index is $\alpha=l ; \zeta$ is used below for longitudinal and transverse modes) in terms of the phonon operators $b_{\mathbf{k}}$ and $b_{\mathbf{k}}^{\dagger}$ is given by [30-32]

$$
u_{\mathrm{ph}}^{\mathbf{k} \alpha}(\mathbf{r})=i \sum_{\alpha=l, \zeta} \sqrt{\frac{\hbar}{2 \rho A_{r} \omega_{\mathbf{k} \alpha}}}|\mathbf{k}| \Xi_{q j} e^{i \mathbf{k} \cdot \mathbf{r}} b_{\mathbf{k} \alpha}+\text { H.c. },
$$

where $\rho$ is the crystal mass density and $A_{r}$ is the area of the graphene sheet. Based on Fermi's golden rule, the phonon- induced p-spin transition rate in GNRs is given by

$$
\frac{1}{T_{1}}=\frac{2 \pi V}{\hbar} \int \frac{d^{3} \mathbf{k}}{(2 \pi)^{3}} \sum_{\alpha=l, \zeta}|M(\mathbf{k} \alpha)|^{2} \delta\left(\hbar s_{\alpha} \mathbf{k}-\varepsilon_{f}+\varepsilon_{i}\right),
$$

where $T_{1}$ is the p-spin lifetime, $s_{l}$ and $s_{\zeta}$ are the longitudinal and transverse acoustic phonon velocities, and $V=A_{r} t$ is the volume. The matrix element $M(\mathbf{k} \alpha)=\left\langle\psi_{i}\left|u_{\mathrm{ph}}^{\mathbf{k} \alpha}(\mathbf{r})\right| \psi_{f}\right\rangle$ with the emission of one phonon $\mathbf{k} \alpha$ has been calculated perturbatively [9]. Here $\left|\psi_{i}\right\rangle$ and $\left|\psi_{f}\right\rangle$ correspond to the initial and finial states of the Hamiltonian $H$. Since we only apply tensile edge stress along $\mathrm{x}$-direction, only longitudinal phonon modes can be utilized to estimate relaxation rate. Based on the second-order nondegenerate perturbation theory, after long algebraic transformations, we have the final result for the orbital relaxation:

$$
\frac{1}{T_{1}}=\frac{\Xi_{0}^{2} t(n+1)}{12 \pi \hbar^{5} \rho m_{0} \omega_{0} s_{l}^{7}}\left(\varepsilon_{f}-\varepsilon_{i}\right)^{5} .
$$

In (20), under the dipole approximation, we write the matrix elements as

$$
|M(\mathbf{k} \alpha)|^{2}=\frac{\hbar^{2} \Xi_{0}^{2} k^{4}(n+1)}{4 \rho \omega_{k l} m_{0} \omega_{0}} \cos ^{2} \phi \sin ^{2} \theta .
$$

To investigate the $\mathrm{p}$-spin flip time, we consider the phonon interaction with (6) and write the matrix elements of (19) as

$$
\begin{aligned}
M(\mathbf{k} \alpha)= & \sum_{n \neq m}\left[\frac{\left\langle m\left|u_{\mathrm{ph}}^{\mathbf{k} \alpha}(\mathbf{r})\right| n\right\rangle\left\langle n \downarrow\left|\tilde{H}_{0}\right| m \uparrow\right\rangle}{\tilde{\varepsilon}_{m}-\tilde{\varepsilon}_{n}-\Delta q^{2} \hbar(m+n+1) / 4 m_{0} \omega_{0}}\right. \\
& \left.+\frac{\left\langle m \downarrow\left|\tilde{H}_{0}\right| n \uparrow\right\rangle\left\langle n\left|u_{\mathrm{ph}}^{\mathbf{k} \alpha}(\mathbf{r})\right| m\right\rangle}{\tilde{\varepsilon}_{m}-\tilde{\varepsilon}_{n}+\Delta q^{2} \hbar(m+n+1) / 4 m_{0} \omega_{0}}\right] .
\end{aligned}
$$

For $m=0$ and $n=1$, we find $\Delta q^{2} \hbar / 2 m_{0} \omega_{0} \ll\left(\tilde{\varepsilon}_{1}-\tilde{\varepsilon}_{0}\right)$. Note that the third term on the right-hand side of (17) is smaller than the second term, which is also ignored in the denominator of (22). Thus the p-spin flip rate between these two lowest states at the Dirac point can be written as

$$
\begin{aligned}
\frac{1}{T_{1}}= & \frac{\Xi_{0}^{2} v_{F}^{2} t}{6 \pi \hbar^{2} \rho m_{0}^{2} \omega_{0}^{2} s_{l}^{7}\left(\tilde{\varepsilon}_{0}-\tilde{\varepsilon}_{1}\right)^{2}}\left(\frac{\Delta q^{2} \hbar}{4 m_{0} \omega_{0}}\right)^{5} \\
& \times\left(1-\frac{q^{2} \hbar}{8 m_{0} \omega_{0}}\right)^{5}\left(\frac{\beta \tau_{e} q}{C_{11} t a}\right)^{2} \\
& \times\left(1-\frac{q^{2} \hbar}{2 m_{0} \omega_{0}}+\frac{q^{4} \hbar^{2}}{16 m_{0}^{2} \omega_{0}^{2}}\right) .
\end{aligned}
$$

In $\tilde{\varepsilon}_{n}$ of (14), we find that $m_{0} v_{F}^{2} \hbar \omega_{0} / A$ is greater than unity for sublattice B and smaller than unity for sublattice A. Thus, we expect different $\mathrm{p}$-spin flip behaviors for sublattices $\mathrm{B}$ and $\mathrm{A}$, which can be considered one of the central results of this paper. We can also express (23) in terms of the control parameters, $\tau_{e}$, and width of the GNRs, $L$, as follows.

For sublattice A

$$
\begin{aligned}
\frac{1}{T_{1}}= & -\frac{\kappa}{192}(1+\chi)\left(1-\gamma+\frac{\gamma^{2}}{4}\right)\left(1-\frac{\gamma}{4}\right)^{5} \\
& \times\left(1+\frac{9}{2} \xi+\frac{91}{8} \xi^{2}+o\left(\xi^{3}\right)\right) \\
& \times\left(1+2 \delta-\delta^{2} / 4+o\left(\tilde{\delta}^{3}\right)\right),
\end{aligned}
$$




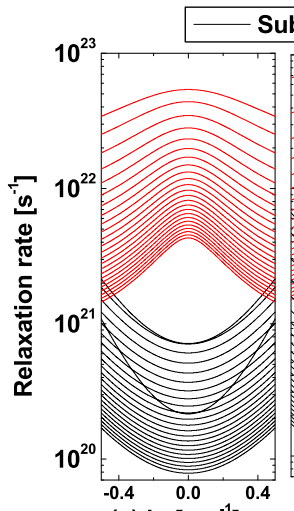

(a) $k_{y}\left[n^{-1}\right]$

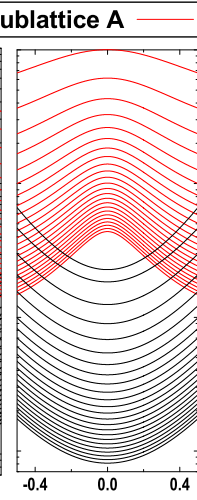

(b) $k_{y}\left[n^{-1}\right]$

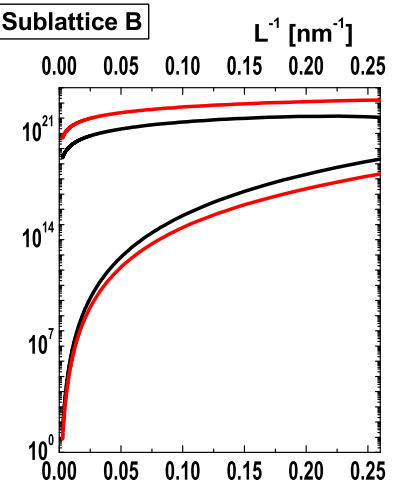

(c) $B_{0}\left(2.3276 \times 10^{4}\right)[T]$

FIG. 3. Orbital relaxation (a) between state $|1 \uparrow\rangle$ and state $|0 \uparrow\rangle$ and (b) between state $|1 \downarrow\rangle$ and state $|0 \downarrow\rangle$. Here we chose $L=$ $3 \sqrt{3} a N$ with $N=8,12, \ldots, 100$ and $\tau_{e}=45 \mathrm{eV} / \mathrm{nm}$. (c) The pspin relaxation $(|0 \downarrow\rangle \rightarrow|0 \uparrow\rangle)$ and orbital relaxation $(|1 \downarrow\rangle \rightarrow \mid 0 \downarrow$ 〉) obtained from (23) and (20) in strained GNRs at the Dirac point. Here we chose $\tau_{e}=100 \mathrm{eV} / \mathrm{nm}$. Other parameters were chosen as $\rho=7.6 \times 10^{-7} \mathrm{~kg} / \mathrm{m}^{2}, \Xi_{0}=6.8 \mathrm{eV}, s_{l}=\sqrt{C_{11} / \rho}$, and $\lambda=2 L$.

and for sublattice B

$$
\begin{aligned}
\frac{1}{T_{1}}= & -\frac{\kappa}{384(2-\sqrt{3})}\left(1-\gamma+\frac{\gamma^{2}}{4}\right)\left(1-\frac{\gamma}{4}\right)^{5} \\
& \times\left(1-4 \xi+10 \xi^{2}+o\left(\xi^{3}\right)\right) \\
& \times\left(1-\frac{3-\sqrt{3}}{3(\sqrt{3}-1)} \delta^{-1}-o\left(\tilde{\delta}^{2}\right)\right)
\end{aligned}
$$

where

$$
\begin{aligned}
& \kappa=\frac{\pi^{13} \Xi_{0}^{2} g_{0}^{5} \mu_{B}^{5} t^{2} a}{\rho \hbar s_{l}^{7} e^{5} \beta \tau_{e} L^{9}}, \quad \chi=\frac{\pi^{3} g_{0}^{2} \mu_{B}^{2} \beta \tau_{e}}{L a C_{11} t e^{2} v_{F}^{2}}, \\
& \delta=\frac{1}{A} m_{0} v_{F}^{2} \hbar \omega_{0}, \quad \xi=\frac{\pi^{3} g_{0}^{2} \mu_{B}^{2} \beta \tau_{e}}{L a C_{11} t e^{2} v_{F}^{2}}, \quad \gamma=\frac{q^{2} \hbar}{2 m_{0} \omega_{0}} .
\end{aligned}
$$

Since $\Delta=-g_{0} \mu_{B} B_{0}$ and $g_{0} \approx-2$, expressions (23)-(25) now have positive values. Evidently, the relaxation rate vanishes like $\tau_{e}^{-1}$ and $L^{-9}$. In Figs. 3(a) and 3(b), we plot the orbital relaxation rate mediated by in-plane phonon modes vs $k_{y}$. Here we clearly see that the orbital p-spin relaxation rates for sublattices A and B are different because the energy bands of sublattices A and B of strained GNRs are not symmetric [see Eq. (10)]. In Fig. 3(c), we plot both the p-spin and the orbital relaxation rates mediated by in-plane phonon modes vs the induced pseudomagnetic field due to ripple waves and the widths of the GNRs. We clearly see that the p-spin relaxation rate for larger ribbon widths vanishes like $L^{-9}$ or $B_{0}^{5}$ [see Eqs. (23)-(25)]. Note that the values of the induced pseudomagnetic fields due to applied tensile edge stress are reduced with increasing widths of GNRs $\left(B_{0} \propto 1 / L\right)$, which is also reflected in Fig. 3(c). An enhancement of the relaxation rate of electrons in sublattice A of GNRs is observed. An enhancement of the $\mathrm{p}$-spin relaxation rate decreases the decoherence time. Thus, designing straintronic devices for application in quantum information processing from highly strained GNRs of sublattice A may not be as suitable as that

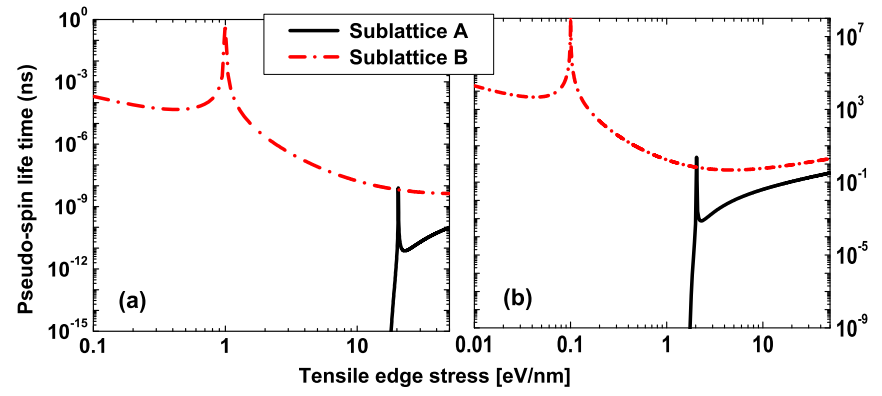

FIG. 4. Pseudospin lifetime $(|0 \downarrow\rangle \rightarrow|0 \uparrow\rangle)$ vs applied tensile edge stress $\tau_{e}$ in strained GNRs at the Dirac point for $L=3 \sqrt{3} a N$ with $N=7$ (left) and $N=70$ (right). Other parameters are the same as in Fig. 3.

from the corresponding GNRs of sublattice B under similar conditions.

In Fig. 4, we plot the p-spin lifetime vs the applied tensile edge stress. Here we find resonant peaks in the manipulation of the phonon-mediated p-spin lifetime with externally applied tensile edge stress. This is due to the fact that the vanishing p-spin splitting energy difference induces a negligible phonon density of states, which provides a vanishing phonon-mediated p-spin transition rate. The exact ideal location of the peak point in the cusp-like structure of the p-spin lifetime can be found from this expression, $\varepsilon_{0 \uparrow}-\varepsilon_{0 \downarrow}=0$. This condition provides us an expression which is quadratic for the applied tensile stress, $\tau_{e}$, and can be written as

$$
\left(\frac{\beta}{C_{11} t a}\right)^{2} \tau_{e}^{2}-\left(\frac{\beta q}{2 C_{11} t a}\right) \tau_{e}-\frac{q^{2}}{64}=0
$$

Thus, the solution of (26) is written as

$$
\tau_{e}=\left(\frac{\sqrt{5} \mp 2}{8}\right)\left(\frac{\pi C_{11} t a}{\beta L}\right)
$$

From Eq. (27), it is clear that $\tau_{e}$ is inversely proportional to the graphene nanoribbon width. This means that the resonant peaks in the p-spin lifetime of GNRs due to applied tensile edge stress for larger GNR widths can be observed at lower values of applied tensile edge stress, which is also reflected in Fig. 4(b). Also, Eq. (27) tells us that the resonant peaks in the p-spin lifetime can be observed at lower values of the applied tensile edge stress for sublattice B compared to sublattice A. Note that the $\mp$ in Eq. (27) corresponds to sublattices B and A, respectively [also see Eq. (11)]. For example, for the parameters chosen in Fig. 4(a) and utilizing Eq. (27), we find the ideal location of the resonant peaks at $\tau_{e} \approx 18 \mathrm{eV} / \mathrm{nm}$ for sublattice $\mathrm{A}$ and $\tau_{e} \approx 1 \mathrm{eV} / \mathrm{nm}$ for sublattice $\mathrm{B}$, which is in agreement with Fig. 4(a). Similarly, by utilizing Eq. (27) and choosing the parameters from Fig. 4(b), we find the resonant peaks at $\tau_{e} \approx 1.8 \mathrm{eV} / \mathrm{nm}$ and $\tau_{e} \approx 0.1 \mathrm{eV} / \mathrm{nm}$ for sublattices $\mathrm{A}$ and $\mathrm{B}$, respectively. In other words, tuning of the resonant peaks of the in-plane phonon-mediated $\mathrm{p}$-spin lifetime in GNRs of sublattice A extends to higher values of the tensile edge stress. 


\section{CONCLUSION}

We have shown that a giant ripple-induced Zeeman p-spin splitting energy can be observed for smaller widths of strained graphene nanoribbons, which may provide evidence of broken p-spin symmetry of degeneracy. By considering in-plane phonon modes in a relaxed-shape armchair GNR, we have shown that highly asymmetric p-spin relaxation behaviors are observed in GNR sublattices A and B. In particular, we have demonstrated that the resonant peak in the p-spin lifetime mediated by the in-plane phonon mode extends to higher values of the applied tensile edge stress for sublattice A than for sublattice B of the GNRs. We have also shown that such resonant peaks can be observed at lower values of the applied tensile edge stress for larger ribbon widths. The resonant peaks can be seen due to the vanishing $\mathrm{p}$-spin splitting energy difference, which induces a negligible phonon density of states and provides a vanishing phonon-mediated p-spin transition rate. Since we have only considered the internal magnetic fields induced by ripple waves, without considering any external source of magnetic fields in the band-structure calculation and estimation of the p-spin lifetime in GNRs, our study may be of special interest for designing optoelectronic devices in straintronics and quantum information processing.

\section{ACKNOWLEDGMENTS}

R.M. was supported by the Natural Sciences and Engineering Research Council (NSERC) of Canada, the Canada Research Chair (CRC) program, and the Bizkaia Talent Grant under the Basque Government through the BERC 2014-2017 program, as well as Spanish Ministry of Economy and Competitiveness MINECO: BCAM Severo Ochoa excellence accreditation SEV-2013-0323.
[1] S. Das Sarma, S. Adam, E. H. Hwang, and E. Rossi, Rev. Mod. Phys. 83, 407 (2011).

[2] A. H. Castro Neto, F. Guinea, N. M. R. Peres, K. S. Novoselov, and A. K. Geim, Rev. Mod. Phys. 81, 109 (2009).

[3] K. S. Novoselov, A. K. Geim, S. V. Morozov, D. Jiang, M. I. Katsnelson, I. V. Grigorieva, S. V. Dubonos, and A. A. Firsov, Nature 438, 197 (2005).

[4] K. S. Novoselov, D. Jiang, F. Schedin, T. J. Booth, V. V. Khotkevich, S. V. Morozov, and A. K. Geim, Proc. Natl. Acad. Sci. USA 102, 10451 (2005).

[5] K. S. Novoselov, A. K. Geim, S. V. Morozov, D. Jiang, Y. Zhang, S. V. Dubonos, I. V. Grigorieva, and A. A. Firsov, Science 306, 666 (2004).

[6] N. Savage, Nature 483, S30 (2012).

[7] F. Bonaccorso, Z. Sun, T. Hasan, and A. C. Ferrari, Nat. Photon. 4, 611 (2010).

[8] W. Han, R. K. Kawakami, M. Gmitra, and J. Fabian, Nat. Nano 9, 794 (2014).

[9] M. Droth and G. Burkard, Phys. Rev. B 87, 205432 (2013).

[10] S. Prabhakar, R. Melnik, L. L. Bonilla, and J. E. Raynolds, Appl. Phys. Lett. 103, 233112 (2013).

[11] N. Levy, S. A. Burke, K. L. Meaker, M. Panlasigui, A. Zettl, F. Guinea, A. H. C. Neto, and M. F. Crommie, Science 329, 544 (2010).

[12] A. Fasolino, J. H. Los, and M. I. Katsnelson, Nat. Mater. 6, 858 (2007).

[13] J. Moser, A. Verdaguer, D. Jimenez, A. Barreiro, and A. Bachtold, Appl. Phys. Lett. 92, 123507 (2008).

[14] S. Prabhakar, R. Melnik, L. L. Bonilla, and S. Badu, Phys. Rev. B 90, 205418 (2014).

[15] S. Prabhakar and R. Melnik, J. Phys.: Condens. Matter 27, 435801 (2015).

[16] L. L. Bonilla, A. Carpio, C. Gong, and J. H. Warner, Phys. Rev. B 92, 155417 (2015).
[17] L. L. Bonilla and A. Carpio, Science 337, 161 (2012).

[18] N. N. Klimov, S. Jung, S. Zhu, T. Li, C. A. Wright, S. D. Solares, D. B. Newell, N. B. Zhitenev, and J. A. Stroscio, Science 336, 1557 (2012).

[19] S. Zhu, Y. Huang, N. N. Klimov, D. B. Newell, N. B. Zhitenev, J. A. Stroscio, S. D. Solares, and T. Li, Phys. Rev. B 90, 075426 (2014).

[20] W.-Y. He, Y. Su, M. Yang, and L. He, Phys. Rev. B 89, 125418 (2014).

[21] C. Lee, X. Wei, J. W. Kysar, and J. Hone, Science 321, 385 (2008).

[22] H. Suzuura and T. Ando, Phys. Rev. B 65, 235412 (2002).

[23] M. Neek-Amal and F. M. Peeters, Phys. Rev. B 82, 085432 (2010).

[24] D. Moldovan, M. Ramezani Masir, and F. M. Peeters, Phys. Rev. B 88, 035446 (2013).

[25] L. D. Landau and E. M. Lifshitz, Theory of Elasticity (Pergamon Press, London, 1970).

[26] A. Carpio and L. L. Bonilla, Phys. Rev. B 78, 085406 (2008).

[27] L. Meng, W.-Y. He, H. Zheng, M. Liu, H. Yan, W. Yan, Z.-D. Chu, K. Bai, R.-F. Dou, Y. Zhang, Z. Liu, J.-C. Nie, and L. He, Phys. Rev. B 87, 205405 (2013).

[28] The choice of vetor potential matches Ref. [22] for armchair graphene nanorobbons where one has to consider chiral angle $\eta=\pi / 6$ (see Eq. (4.3) in Ref. [22]).

[29] J. Balakrishnan, G. K. W. Koon, M. Jaiswal, A. H. Castro Neto, and B. Ozyilmaz, Nat. Phys. 9, 284 (2013).

[30] C. Kittel, Introduction to Solid State Physics, 8th ed. (John Wiley $\&$ Sons, New York, 2012).

[31] K. H. Michel, S. Costamagna, and F. M. Peeters, Phys. Rev. B 91, 134302 (2015).

[32] K. S. Tikhonov, W. L. Z. Zhao, and A. M. Finkel'stein, Phys. Rev. Lett. 113, 076601 (2014). 